\title{
Microbiological Evaluation of Osteomyelitis with A Special Reference to Antibiotic Sensitivity Pattern of Isolates from A Tertiary Care Hospital
}

\author{
Barnini Banerjee $^{1}$ (D) Smitha S. Bhat ${ }^{1}$, Chiranjay Mukhopadhyay ${ }^{1 *}$ (D) \\ and Anil K. Bhat ${ }^{2}$ iD
}

${ }^{1}$ Department of Microbiology, Kasturba Medical College, Manipal, Manipal Academy of Higher Education, Manipal - 576104, India. ${ }^{2}$ Department of Orthopedics, Kasturba Medical College, Manipal, Manipal Academy of Higher Education, Manipal - 576104, India.

\begin{abstract}
Epidemiological change in osteomyelitis had been observed for the past few years with a significant rise of chronic osteomyelitis by multidrug-resistant organisms (MDROs). It complicates antibiotic selection, and if not managed adequately, it results in amputation, sepsis, or death. This study was conducted for one year in a tertiary care hospital to identify the etiological agents and their response to antibiotics for the development of adequate management policy of osteomyelitis. Fifty seven patients were analysed, $\&$ the variables examined include epidemiological characteristics, risk factors, radiographic changes, histopathological features. Etiological agents were identified, and antimicrobial susceptibility testing was performed by an automated system. About $73.7 \%$ of the cases presented with chronic osteomyelitis in the age group of $31-40$ years (29.3\%). In 53 patients, it resulted from contagious microbial seeding. The most common site of infection was the metatarsal bones of the foot $(38.6 \%)$. The common predisposing factor was Diabetes (36.8\%). The most common causative organism was Staphylococcus aureus (46.9\%). Methicillin resistance was 41.9\%. Among Gram-negative isolates, Enterobacterales group predominated (22.7\%), 42.9\% of Gram-negatives were multidrug -resistant (MDR). In our study, we found an increased prevalence of chronic osteomyelitis of the extremities of the lower limb instead of acute hematogenous infection of the long bones. Most of them resulted from trauma or after fracture fixation device implantation or as a sequela of diabetic foot. Fourty percent of the etiological agents in our study were either methicillin resistant S. aureus (MRSA) or MDR Gram-negatives. This finding is challenging for the clinician to select the appropriate treatment option, reduce the risk of recurrence and improve the quality of life of the patients.
\end{abstract}

Keywords: Chronic, Osteomyelitis, Multi drug resistance, Implant

*Correspondence: chiranjay@gmail.com; + 918202933529

(Received: July 22, 2019; accepted: January 27, 2020)

Citation: Barnini Banerjee, Smitha S. Bhat, Chiranjay Mukhopadhyay and Anil K. Bhat, Microbiological Evaluation of Osteomyelitis with A Special Reference to Antibiotic Sensitivity Pattern of Isolates from A Tertiary Care Hospital, J. Pure Appl. Microbiol., 2020; 14(1):485-490. https://doi.org/10.22207/JPAM.14.1.50

(C) The Author(s) 2020. Open Access. This article is distributed under the terms of the Creative Commons Attribution 4.0 International License which permits unrestricted use, sharing, distribution, and reproduction in any medium, provided you give appropriate credit to the original author(s) and the source, provide a link to the Creative Commons license, and indicate if changes were made. 


\section{INTRODUCTION}

Osteomyelitis (OM) is a one of the most challenging bone and joint infection till the present day due to its heterogeneity. Though Staphylococcus is the classical culprit of OM, Gramnegative bacilli are also gaining recognition as the etiological agents. In the modern era, increase in Gram-negatives probably due to increase use of fracture fixation devices in orthopaedic surgeries, especially in open fracture cases after high energy trauma. Their contribution to diabetic foot osteomyelitis also not less than Gram-positives. The real matter of concern nowadays is multidrug resistance among both Gram-negative \& Gram-positive organisms. Among Gram-negative bacilli, resistance to carbapenems is yet another concern. Carbapenem resistant not even unusual in the present day. The reason for the prevalence of multidrug resistance organisms is believed to be the virulence factors retained by this microorganism, which enable it to adhere and invade host tissues and evade immune defences and antimicrobials. Biofilm formation is the primary pathogenic mechanism leading to the chronicity and irreducibility of infections. Their presence delays the drug penetration to the bone, promote the antimicrobial resistance and increase the chronicity.

The frequency of isolation of organisms and antimicrobial resistance pattern of the different pathogens always vary in a different geographical region. Hence this study will help to develop the antimicrobial policy for osteomyelitis, which can ultimately help the clinician in the management of osteomyelitis. Present study is done to explore clinical characteristics, bacterial aetiology, and antimicrobial susceptibility pattern of the aerobic bacteria causing OM.

\section{MATERIAL AND METHODS Study design}

A total of 57 adult patients who were diagnosed and treated for osteomyelitis in our hospital during one year were included in this study. Clinical and other laboratory data of the patients were reviewed further. We excluded the cases of tubercular or anaerobic bacterial etiology. Infections caused by more than two bacteria were also excluded.

\section{Definitions}

a. Co-infections: When more than one organism isolated from the clinical specimen of the same patient at the same time.

b. Multidrug resistant organisms (MDROs): Microorganisms which were non-susceptible to $\geq 1$ agent in $\geq 3$ antimicrobial categories.

c. Osteomyelitis: All the osteomyelitis cases were diagnosed based on clinical presentation, histopathological findings and radiological picture. According to the time period between diagnosis and symptom onset, osteomyelitis was classified as acute ( $<2$ weeks), sub-acute ( 2 weeks -3 months), or chronic ( $>3$ months).

\section{Microbiological workup}

Clinical specimens including deep wound swab, tissue or pus were collected and cultured on $5 \%$ sheep blood agar and MacConkey agar. The growth in the culture media was identified by the Matrix Assisted Laser Desorption Ionization TimeOf-Flight (MALDI-TOF) VITEK ${ }^{\circledR}$ MS. Antimicrobial susceptibility testing was done by Vitek ${ }^{\circledR} 2$ system (BioMerieux, Inc, Durham, NC).

\section{RESULTS}

\section{Demographic \& clinical characteristics}

Of the total 57 patients included in the study, 42 (73.7\%) presented with chronic osteomyelitis having pain $(54 / 68,79.4 \%)$ \& discharging sinus $(52 / 68,76.5 \%)$. In acute cases, swelling was there along with pain instead of discharging sinuses. The median length of their hospital stay was 12.5 days (range-3-30days). The mean (SD) age of the patients was 46.4 years $( \pm 14.12)$, and $78.9 \%(45 / 57)$ of the patients were male. Maximum cases of chronic osteomyelitis were observed in the age group of 31-40 years $(29.3 \%, 12 / 41)$. The significant correlation observed with the distribution of different types of osteomyelitis at various age group ( $p$ value-.025). OM was universal in the lower limb, and the most common site of infection was the metatarsal bones of the foot $(22 / 57,38.6 \%)$ followed by tibia $(13 / 57,22.8 \%)$. Out of 57 cases, $53(92.9 \%)$ patients had contagious osteomyelitis resulted from direct spread from nearby ulceration $(18 / 57,31.6 \%)$, post-traumatic $(16 / 57,28 \%)$ and postoperatively after fixation of the fracture 
with devices (19/57, 33.3\%) (Table 1). The major underlying predisposing factor was diabetes $(36.8 \%)$ and presence of fracture fixation devices (33.3\%). 34.1\% (14/41) of patients developed chronic OM followed by fracture fixation devices.

\section{Microbiological Characteristics}

A total of 66 microbial agents isolated from 57 patients. 45 (68.1\%) of them were Gram positive (GP), and 21 of them were Gram negative (31.8\%) (GN) bacteria. Out of 45 GP bacteria, 9 of them caused acute OM (20\%) and 36 of them caused chronic OM (80\%). Among $21 \mathrm{GN}$ bacteria, $8(38.1 \%)$ contributed to acute OM and 13 (61.9\%)

Table 1. Demographic \& Clinical characteristics

\begin{tabular}{lcc}
\hline Age group & $\begin{array}{c}\text { Acute OM } \\
\mathrm{n}=15(\%)\end{array}$ & $\begin{array}{c}\text { Chronic OM } \\
\mathrm{n}=42(\%)\end{array}$ \\
\hline $\begin{array}{l}\text { 20-40 years } \\
19(45.2)\end{array}$ & $03(20)$ & \\
$41-60$ years & $09(60)$ & $18(42.9)$ \\
$61-80$ years & $03(20)$ & $05(11.9)$ \\
$\begin{array}{l}\text { Gender } \\
\text { Male }\end{array}$ & $11(73.3)$ & $34(80.9)$ \\
$\begin{array}{l}\text { Female } \\
\text { Affected segment }\end{array}$ & $04(26.7)$ & $08(19.1)$ \\
$\begin{array}{l}\text { Upper limb } \\
\text { Lower Limb }\end{array}$ & $02(13.3)$ & $02(4.7)$ \\
$\begin{array}{l}\text { Mechanism of OM } \\
\text { Contagious OM }\end{array}$ & $13(86.7)$ & $40(95.3)$ \\
Hematogenous OM & $15(100)$ & $38(90.4)$ \\
\hline
\end{tabular}

of them led to chronic OM. 24.4\% Of Gram positive bacteria and $23.8 \%$ of GNB caused OM post-fracture fixation with devices. Most common causative organisms were Staphylococcus aureus (31/66, 46.9\%) followed by Enterobacteriaceae group (15/66, 22.7\%) (Table 2). Out of 31 isolates of S. aureus, 13 (41.9\%) were Methicillin-resistant (MRSA). They showed maximum no. of resistance to ciprofloxacin (24/31, 77.1\%). $100 \%$ of MRSA strains were sensitive to vancomycin. Large number of susceptibility was also observed for cotrimoxazole (80.6\%) \& tetracycline (30/31, 96.8\%) (Figure 5). All beta-hemolytic Streptococci (BHS) were sensitive to a penicillin (100\%) \& ceftriaxone $(100 \%)$ and the Enterococci were susceptible to penicillin \& high-level gentamicin. Out of 21

Table 2. Etiological agents of OM

\begin{tabular}{lc}
\hline Etiological agents & Total Number (\%) \\
\hline S. aureus & $31(46.9 \%)$ \\
Beta-haemolytic & $11(16.7)$ \\
Streptococcus spp & \\
Enterococcus spp & $03(4.5)$ \\
E. coli & $03(4.5)$ \\
Klebsiella pneumoniae & $04(6.1)$ \\
Citrobacter freundii & $03(4.5)$ \\
Enterobacter cloacae & $03(4.5)$ \\
Proteus vulgaris & $01(1.5)$ \\
Serratia marcesens & $01(1.5)$ \\
Pseudomonas aeruginosa & $06(9.1)$ \\
\hline
\end{tabular}

Table 3. Antibiotic susceptibility of Gram positive bacteria [Number (\%) of sensitive strain]

\begin{tabular}{lccc}
\hline Antibiotics & $\begin{array}{c}\text { S. aureus } \\
\mathrm{N}=31\end{array}$ & $\begin{array}{c}\text { Beta-hemolytic } \\
\text { Streptococcus } \\
\text { spp (N=11) }\end{array}$ & $\begin{array}{c}\text { Enterococcus } \\
\text { spp (N=3) }\end{array}$ \\
\hline Cloxacillin & $18(58 \%)$ & - & - \\
Penicillin & $11(35.5 \%)$ & $11(100 \%)$ & $3(100 \%)$ \\
Ciprofloxacin & $7(22.6 \%)$ & $11(100 \%)$ & $01(33.3 \%)$ \\
Co-trimoxazole & $25(80.6 \%)$ & $9(81.8 \%)$ & - \\
Erythromycin & $19(61.3 \%)$ & $07(63.6 \%)$ & $01(33.3 \%)$ \\
Gentamycin & $24(77.4 \%)$ & $11(100 \%)$ & $3(100 \%)$ \\
Tetracycline & $30(96.8 \%)$ & $3(27.3 \%)$ & $3(100 \%)$ \\
Clindamycin & $28(90.3 \%)$ & $9(81.8 \%)$ & - \\
Vancomycin & $31(100 \%)$ & $11(100 \%)$ & $3(100 \%$ \\
Linezolid & $31(100 \%)$ & $11(100 \%)$ & $3(100 \%)$ \\
Teicoplanin & $31(100 \%)$ & $11(100 \%)$ & $3(100 \%)$ \\
Ceftriaxone & - & $11(100 \%)$ & - \\
Levofloxacin & - & $8(72.7 \%)$ & -
\end{tabular}


Table 4. Antibiotic susceptibility of Gram negative bacteria [number(\%) of sensitive strain]

\begin{tabular}{lccccc}
\hline Antibiotics & $\begin{array}{c}\text { E.coli } \\
\mathrm{N}=3\end{array}$ & $\begin{array}{c}\text { Klebsiella } \\
\mathrm{N}=4\end{array}$ & $\begin{array}{c}\text { Enterobacter } \\
\mathrm{N}=3\end{array}$ & $\begin{array}{c}\text { Citrobacter } \\
\mathrm{N}=3\end{array}$ & $\begin{array}{c}\text { Pseudomonas } \\
\mathrm{N}=6\end{array}$ \\
\hline Ampicillin & $1(33.3 \%)$ & - & - & $1(33.3 \%)$ & - \\
Amoxy-clav & $1(33.3 \%)$ & - & - & $2(66.7 \%)$ & - \\
Ciprofloxacin & $1(33.3 \%)$ & $1(25 \%)$ & $3(100 \%)$ & $2(66.7 \%)$ & $4(66.7 \%)$ \\
Co-trimoxazole & $1(33.3 \%)$ & $1(25 \%)$ & $2(66.7 \%)$ & $2(66.7 \%)$ & - \\
Amikacin & $3(100 \%)$ & $3(75 \%)$ & $3(100 \%)$ & $3(100 \%)$ & $4(66.7 \%)$ \\
Gentamycin & $1(33.3 \%)$ & $3(75 \%)$ & $3(100 \%)$ & $2(66.7 \%)$ & $4(66.7 \%)$ \\
Ceftriaxone & - & - & $3(100 \%)$ & $2(66.7 \%)$ & - \\
Cefuroxime & - & - & - & $1(33.3 \%)$ & - \\
Cef-sulbactam & $3(100 \%)$ & $1(25 \%)$ & $3(100 \%)$ & $3(33.3 \%)$ & $4(66.7 \%)$ \\
Pip-tazobactam & $3(100 \%)$ & $1(25 \%)$ & $3(100 \%)$ & $2(66.7 \%)$ & $05(83.3 \%)$ \\
Imipenem & $3(100 \%)$ & $2(50 \%)$ & $3(100 \%)$ & $3(100 \%)$ & $5(83.3 \%)$ \\
Cefepime & $3(100 \%)$ & $2(50 \%)$ & $3(100 \%)$ & $3(100 \%)$ & $5(83.3 \%)$ \\
Ceftazidime & - & - & - & - & $4(66.7 \%)$ \\
\hline
\end{tabular}

Gram negative isolates, 9 (42.9\%) were multidrugresistant (MDR). Most common MDR organism was K. pneumoniae (3/4, 75\%). 13.3\% (2/15) of Enterobacterales \& $16.7 \%(1 / 6)$ of Pseudomonas aeruginosa were carbapenem-resistant. More than $80 \%$ of Enterobacterales were resistant to ampicillin, amoxy-clavulanic acid \& first, second \& third generation of cephalosporin (Table $3 \& 4$ ). Clinical Outcome

Out of 57 patients, 18 of them not improved clinically and radiologically. They revisited the hospital within six months. Of 18 patients, 11 of them had infection due to MDR organisms (61.1\%).Twelve of them (21\%) had undergone amputation. Out of 16 patient with an implant, 6 of them had implant failure (37.5\%).

\section{DISCUSSION}

Osteomyelitis is an acute or chronic inflammation of the bone caused by an infectious organism and is a challenging clinical problem. It is essential to identify the etiological agents to treat the infection.

The present study, after investigating a cohort of 57 patients, found that the mean age of patients was 46.4 years $\& 78.9 \%$ of patients were male. Similar results were found by Jiang $\mathrm{N}$ et al. where they found the median age to be 42 years $\&$ increased male predilection ${ }^{1}$.

We observed out of 57 cases, $73.7 \%$ of them had chronic osteomyelitis and a significant number of them were in the age group of 31-60 years (30/42, 71.4\%). Similarly, Prieto-Pérez et al. ${ }^{2}$ found more number of chronic osteomyelitis cases among patients within the age group of 41- 60 years. The incidence of acute osteomyelitis was only $26.3 \%$. Acute osteomyelitis is more common in children less than five years of age due to bacteremic seeding of bone ${ }^{18}$. As we have not included children in our study, so less number of acute osteomyelitis had been depicted. Chronic osteomyelitis is much less observed among children ${ }^{3}$.

Several etio-pathogenic mechanisms for osteomyelitis were reported in the past. Infection can occur secondary to a contiguous-focus, by direct contamination after trauma or surgery or infections originating from the bloodstream ${ }^{4}$. In this study, we found, most of the disease developed by direct inoculation (45/57, 78.9\%) of bacteria or by seeding of them from next focus (31.6\%). Jorge SL et al. also depicted that the incidence of osteomyelitis by direct inoculation has increased over the last decades ${ }^{5}$. This is due to the increased frequency of accidents followed by more use of orthopaedic fixation devices and joint prostheses. Traditional chronic hematogenous osteomyelitis is now supplemented by frequently encountered postop (33.3\%), post-traumatic (28\%) \& implant-related infection $(33.3 \%)^{6}$. The primary underlying predisposing factor was diabetes (36.8\%) for acute \& chronic osteomyelitis 
secondary to contagious soft tissue infection. It has been reported that up to one-half of patients with diabetes develop peripheral neuropathy, which may reduce their awareness of wounds and increase the risk of unrecognized infections.

The metatarsal bones of the foot followed by the tibia and femur were the most commonly affected bones in our study $(38.6 \%, 22.8 \%$ \& $15.9 \%$ respectively). Jiang $\mathrm{N}$ et al..$^{1}$ observed the tibia was the most common site in traumatic osteomyelitis, while the femur and toes were the most common sites in hematogenous and diabetic foot osteomyelitis. We found frequent infection in metatarsal bones as a complication of diabetic foot.

Clinical symptoms of osteomyelitis can be nonspecific and difficult to recognize. Signs and symptoms change depending on the category of infection, organism \& anatomical location of the disease. In our study, the majority of the patients with osteomyelitis presented with pain (79.4\%) \& discharging sinus $(76.5 \%)$. Pain \& discharging sinuses were common clinical presentations in chronic cases, whereas pain \& swelling were frequent in acute cases.

During the study period, a total of 86 samples were collected from 57 patients for microbiological analysis. Out of 57 patients, monomicrobial infection observed among 55.3\% of patients \& co-infection was $42.6 \%$. In our study, the majority of postoperative and posttraumatic infections were caused by two microbes $(12 / 27=44.4 \%)$ similar to Mathewa PG et al. ${ }^{26}$ but higher than Trampuz et al. $(27 \%)^{7}$. Monomicrobial infection by Gram positive bacteria was commonly observed $(9 / 16,56.2 \%)$ in contagious osteomyelitis due to the spread from adjacent ulcer site. This is in contrast to Calhoun JH et al. ${ }^{8}$.

Staphylococcus aureus (46.9\%) was the most frequently isolated bacteria overall similar to Lobati $\mathrm{F}$ et $\mathrm{al}^{9}$. We also had a fair number of betahemolytic Streptococcus spp (20\%) from chronic cases. Generally, they are frequent etiological agent among children, rare in adults ${ }^{10}$.

In view of chronic osteomyelitis, PG Mthethwa et al. ${ }^{11}$ found Enterobacterales as a most common organism (34\%). In contrast, we found $S$. aureus as the most prevalent organism for both acute (46\%) \& chronic infection (50\%) followed by Enterobacterales (18.8\%\& 24\% respectively). Prevalence of $P$. aeruginosa in our study was $9.1 \%$ similar to other study $(11 \%)^{11}$.

Out of 31 isolates of S.aureus, $41.9 \%$ were MRSA with $100 \%$ of them being susceptible to vancomycin. Reason for the high number of MRSA could be that most of our cases were having posttraumatic or post-op infection. They showed high susceptibility for co-trimoxazole (80.6\%) which have been found to achieve bone concentrations at $\sim 50 \%$ of serum $\&$ as effective as vancomycin. Other than co-trimoxazole, clindamycin is another oral agent available against community-acquired MRSA, which reliably penetrates bone at levels of approximately $40 \%-70 \%$ of serum. In our study, susceptibility of clindamycin was $76.9 \%{ }^{12}$. We also found, out of 15 Enterobacterales, more than $80 \%$ of them were ESBL producers, resistant to ampicillin, amoxy-clavulanic acid \& first, second $\&$ third generation of cephalosporins. More than $80 \%$ of the ESBL producers were susceptible to carbapenem, piperacillin-tazobactam \& cefepime similar to Mthethwa PG et al. $60 \%$ of Gram-negatives also showed resistance against fluoroquinolones \& aminoglycosides in contrast to Spellberg et al. ${ }^{12}$. So piperacillin-tazobactam along with clindamycin can be considered for empirical therapy which can be modified later based on culture findings.

$46.7 \%$ of the Enterobacterales were found to be MDR. Most common MDR was K. pneumoniae (75\%) \& $50 \%$ of them were carbapenem resistance. Pseudomonas also showed carbapenem resistance of $16.7 \%$ in contrast to Mthethwa PG et a ${ }^{11}$. We also observed quite a high number of morbidity in our study due to persistence of the MDR organisms (61.1\%). Among the MDROs, MDR MRSA contributed (8/11) $72.7 \%$ whereas MDR GNBs not attributed much (27.3\%). But we did not find any significant correlation $(p=0.5)$ when we compared clinical outcomes between patients with and without MDR organisms.

$21 \%$ of our patient had undergone amputation. Even, out of the 16 patient with an implant, 6 of them had implant failure (37.5\%) within six months.

\section{CONCLUSION}

Osteomyelitis is one of the challenging infections of the bone. Clinical pictures of it changed markedly in the past few decades. In our 
study, we found an increased prevalence of chronic osteomyelitis of the extremities of lower limb instead of acute hematogenous infection of the long bones. Most of them resulted from trauma or post-implant with fracture fixation devices or as a sequela of diabetic foot \& predominated in adult males. Monomicrobial infection was more. S. aureus \& P. aeruginosa was the common Gram positive \& gram negative bacteria. A total of $40 \%$ isolate were either MRSA or MDR Gram negatives. This finding is a matter of concern \& challenging for the clinician in order to select the appropriate treatment option, reduce the risk of recurrence and improve the quality of life of the patients.

\section{ACKNOWLEDGEMENTS}

None.

\section{CONFLICT OF INTEREST}

The authors declare that there is no conflict of interest.

\section{FUNDING}

None.

\section{AUTHORS' CONTRIBUTIONS}

BB \& CM conceptualized the study. SB, $B B$ and $A B$ contributed in data interpretation. SB \& BB compiled information from the results and literature and drafted the manuscript. $C M$ \& $A B$ helped in draft improvisation. $B B, C M$ and $A B$ supervised and reviewed the manuscript. $B B, S B$, $C M$, and $A B$ read and approved the manuscript.

\section{DATA AVAILABILITY}

All datasets generated or analyzed during this study are included in the manuscript.

\section{ETHICS STATEMENT}

This study was conducted after taking the permission from institutional ethical committee.

\section{REFERENCES}

1. Jiang $N$, Ma $Y$, Jiang $Y$, Zhao $X$, Xie G, Hu Y et al. Clinical Characteristics and Treatment of Extremity Chronic Osteomyelitis in Southern China. Medicine, 2015; 94(42): e1874. https://doi.org/10.1097/ MD.0000000000001874

2. Prieto-Perez L, Perez-Tanoira R, Petkova-Saiz E, Perez-Jorge C, Lopez-Rodriguez C, Alvarez-Alvarez B et al. Osteomyelitis: A Descriptive Study. Clin Orthop Surg, 2014; 6(1): 20. https://doi.org/10.4055/ cios.2014.6.1.20

3. Gutierrez K. Bone and joint infections in children. Pediatr Clin North Am, 2005; 52(3): 779-794. https://doi. org/10.1016/j.pcl.2005.02.005

4. Auh JS, Binns HJ, Katz BZ. Retrospective assessment of subacute or chronic osteomyelitis in children and young adults. Clin Pediatr (Phila), 2004; 43(6): 549-555. https://doi.org/10.1177/000992280404300607

5. Waldvogel FA, Medoff G, Swartz MN. Osteomyelitis: a review of clinical features, therapeutic considerations and unusual aspects. N Engl J Med, 1970; 282(4): 198206. https://doi.org/10.1056/NEJM197001222820406

6. Jorge L, Chueire A, Baptista Rossit A. Osteomyelitis: a current challenge. Braz J Infect Dis, 2010; 14(3): 31015.

7. Trampuz A, Zimmerli W. Diagnosis and treatment of infections associated with fracture- fixation devices. Injury, 2006; 37(Suppl 2): 59-66.

8. Calhoun J, Manring M, Shirtliff M. Osteomyelitis of the Long Bones. Semin Plast Surg, 2009; 23(02): 059-072.

9. Lobati F, Herndon B, Bamberger D. Osteomyelitis: Etiology, Diagnosis, Treatment and Outcome in a Public versus a Private Institution. Infection, 2001; 29(6): 333336. https://doi.org/10.1007/s15010-001-1134-6

10. Brady RA, Leid JG, Costerton JW, Shirtliff ME. Osteomyelitis: Clinical overview and mechanisms of infection persistence. Clin Microbiol Newsl, 2006; 28(9): 65-72. https://doi.org/10.1016/j. clinmicnews.2006.04.001

11. Mthethwa P, Marais L. The microbiology of chronic osteomyelitis in a developing world setting. SA orthop, 2017; 16(2). https://doi.org/10.17159/23098309/2017/v16n2a4

12. Spellberg B, Lipsky A. Systemic antibiotic therapy for chronic osteomyelitis in adults clinical infectious diseases. Clin Infect Dis, 2012; 54(3): 393-407. https:// doi.org/10.1093/cid/cir842 